\title{
Removal of Cardiopulmonary Resuscitation Artifacts with an Enhanced Adaptive Filtering Method: An Experimental Trial
}

\author{
Yushun Gong, ${ }^{1}$ Tao Yu, ${ }^{2,3}$ Bihua Chen, ${ }^{1} \mathrm{Mi} \mathrm{He},{ }^{1}$ and Yongqin $\mathrm{Li}^{1,3}$ \\ ${ }^{1}$ School of Biomedical Engineering, Third Military Medical University and Chongqing University, 30 Gaotanyan Main Street, \\ Chongqing 400038, China \\ ${ }^{2}$ Emergency Department, Sun Yat-Sen Memorial Hospital of Sun Yat-Sen University, Guangzhou 510120, China \\ ${ }^{3}$ Institute of Cardiopulmonary Cerebral Resuscitation, Sun Yat-Sen University, Guangzhou 510120, China
}

Correspondence should be addressed to Yongqin Li; leeoken@gmail.com

Received 30 November 2013; Revised 25 February 2014; Accepted 26 February 2014; Published 27 March 2014

Academic Editor: Giuseppe Ristagno

Copyright (C) 2014 Yushun Gong et al. This is an open access article distributed under the Creative Commons Attribution License, which permits unrestricted use, distribution, and reproduction in any medium, provided the original work is properly cited.

Current automated external defibrillators mandate interruptions of chest compression to avoid the effect of artifacts produced by CPR for reliable rhythm analyses. But even seconds of interruption of chest compression during CPR adversely affects the rate of restoration of spontaneous circulation and survival. Numerous digital signal processing techniques have been developed to remove the artifacts or interpret the corrupted ECG with promising result, but the performance is still inadequate, especially for nonshockable rhythms. In the present study, we suppressed the CPR artifacts with an enhanced adaptive filtering method. The performance of the method was evaluated by comparing the sensitivity and specificity for shockable rhythm detection before and after filtering the CPR corrupted ECG signals. The dataset comprised 283 segments of shockable and 280 segments of nonshockable ECG signals during CPR recorded from 22 adult pigs that experienced prolonged cardiac arrest. For the unfiltered signals, the sensitivity and specificity were $99.3 \%$ and $46.8 \%$, respectively. After filtering, a sensitivity of $93.3 \%$ and a specificity of $96.0 \%$ were achieved. This animal trial demonstrated that the enhanced adaptive filtering method could significantly improve the detection of nonshockable rhythms without compromising the ability to detect a shockable rhythm during uninterrupted CPR.

\section{Introduction}

Early defibrillation is critical for the survival of patient who suffered from cardiac arrest $[1,2]$. However, the application of high quality of cardiopulmonary resuscitation (CPR) introduces strong artifact components into the electrocardiogram (ECG) signal, which reduces the accuracy of the shock/nonshock decision of automated external defibrillators (AEDs) [3]. Thus, chest compressions (CC) are mandated to be interrupted in the current AEDs in order to perform a reliable rhythm analysis and provide appropriate defibrillation prompt to the rescuers. But even seconds of interruptions of CC adversely affects the rate of restoration of spontaneous circulation (ROSC) and survival [4]. According to an experimental study, the likelihood of successful resuscitation decreased as much as $50 \%$ with a 20 -second interruption of CC [5]. Actually, clinical studies have also confirmed that longer pauses in CC before and after defibrillator shocks were independently associated with a decrease in survival to hospital discharge $[6,7]$. When the hands-off intervals were minimized, significantly better outcomes were achieved and reported $[8,9]$. Therefore, the latest guidelines from the American Heart Association (AHA) and the European Resuscitation Council (ERC) recommended minimizing these hands-off intervals between compression and shock [10, 11].

If accurate cardiac rhythm analysis can be performed during CPR, these interruptions will be minimized or totally avoided. During the last decade, numerous digital signal processing techniques have been developed to remove the artifacts or interpret CC corrupted ECG during CPR. Sensitivity and specificity are the proportion of correctly identified shockable and nonshockable rhythms, respectively, and are used to evaluate the performance of artifact suppression method. Algorithms removing artifacts using only the ECG signal, including independent component analysis (ICA) [12] and coherent line removal algorithm [13], have improved the 
sensitivity to $99.8 \%$ and the specificity to $83.2 \%$ for detecting a shockable rhythm. Methods filtering the CPR artifact using additional references, such as Gabor multipliers [14], Kalman filter [15], adaptive filter [16-19], and multichannel recursive adaptive matching pursuit (MC-RAMP) filter [20], have improved the sensitivity and specificity to $95.6 \%$ and $90.5 \%$. To identify a shockable rhythm during CPR, Li et al. [21] searched the identifiable components directly in the corrupted ECG signal using morphology consistence evaluation. A sensitivity of $93.3 \%$ and specificity of $88.6 \%$ were reported in a dataset which consisted of 229 victims during out-of-hospital cardiac arrest. Although the sensitivity for detecting a shockable rhythm was significantly improved with the application of these techniques, the specificity was still below the $95 \%$ limit recommended by the AHA task force on AEDs for accurately detecting nonshockable rhythms [22]. Further studies are, therefore, still required to analyze the interaction between the artifact and underlying rhythms and to improve the accuracy of nonshockable rhythm decision $[23,24]$.

In the present study, the effects of CC on signal-to-noise ratio (SNR) at different types of underlying rhythms (ventricular fibrillation (VF), pulseless electrical activity (PEA), and asystole (ASY)) were firstly analyzed in an adult porcine model of prolonged cardiac arrest and CPR. An enhanced adaptive filtering method was then developed to suppress the CPR artifact and evaluated by comparing the sensitivity and specificity for shockable rhythm detection before and after filtering.

\section{Materials and Methods}

2.1. Experiment Procedure and Data Collection. The experimental data were collected from 22 male adult pigs that experienced prolonged cardiac arrest and CPR. The porcine model has been well established to simulate real out-ofhospital scenarios due to the fact that heart size, blood pressure, and heart rate are similar to those in humans [25]. Anesthesia was initiated by intramuscular injection of ketamine $(20 \mathrm{mg} / \mathrm{kg})$ and was completed by ear vein injection of sodium pentobarbital $(30 \mathrm{mg} / \mathrm{kg})$. VF was electrically induced by applying a $5 \mathrm{~mA}$ alternate current through a pacing catheter in the right ventricle. $\mathrm{CPR}$, including $\mathrm{CC}$ and ventilation, was begun after 6 minutes of untreated VF (Group A) in 14 animals [26]. The compression depth (CD) was randomized to either $25 \%$ or $17 \%$ of the anterior posterior diameter of the chest during the first 4 minutes of CPR and $20-25 \%$ after 4 minutes. In another 8 animals with the same weight and chest size, CPR was begun after 11 minutes of untreated VF (Group B). CD was comparable to $20-25 \%$ of the anterior posterior diameter of the chest. For all of the animals, manual CC were performed by two experienced emergency medical doctors at a rate above 100 per minute. The animals were manually ventilated with a bag-valve device during CPR. CC were synchronized to provide a compression/ventilation ratio of $30: 2$ with equal compression-relaxation intervals. After 2 minutes of CC in Group A and 6 minutes of compression in Group B, a defibrillation was attempted with a single $120 \mathrm{~J}$ rectilinear biphasic shock. One dose of epinephrine $\left(30 \mu \mathrm{g} \cdot \mathrm{kg}^{-1}\right)$ was given through the right atrial catheter after 2 minutes of CPR in Group B. CC were immediately resumed followed by ECG rhythm analysis within 5 seconds until confirmation of spontaneous circulation. If spontaneous circulation was not restored, CC were continued for another 2 minutes, after which defibrillation was attempted with another single $120 \mathrm{~J}$ shock. This sequence was repeated for a maximum of 5 cycles.

The ECG, acceleration, and transthoracic impedance (TTI) waveform were continuously measured and recorded through a data acquisition system supported by Windaq hardware/software (Dataq Instruments Inc., Akron, OH, USA) at a sample rate of $300 \mathrm{~Hz}$. During CC, the acceleration and TTI signals also served as feedback to control the compression rate and depth. The ECG was measured from the output of a commercial defibrillator (M-Series, Zoll medical corporation, Chelmsford, MA, USA) with the use of a hard gel type of adult defibrillation/pacing pads (statpadz, Zoll Medical Corporation, Chelmsford, MA, USA) that were applied with an anterior to lateral placement. TTI waveform was recorded through a user designed circuit which was parallelly connected with the defibrillator using a sinusoid-wave excitation current of $2 \mathrm{~mA}$ and $30 \mathrm{kHz}$ across the defibrillation pads. The acceleration signal was recorded from an accelerometer-based handheld CPR device (CPR-Dpadz, Zoll Medical Corporation, Chelmsford, MA, USA) that was placed on the surface of the animal's chest just above the heart and underneath the rescuer's hands during CC.

Data were analyzed offline through user designed software using Matlab (The MathWorks, Inc., Natick, MA, USA). ECG, together with acceleration and TTI signals during CPR, was extracted and annotated from the digitalized experimental records. The CD was calculated from the double integration of acceleration signal. Each segment consisted of 4-second corrupted signal and 3-second artifact-free signal, either during ventilation or during rhythm analysis. These segments were then annotated as VF, PEA, or ASY by an experienced emergency medical doctor. As shown in Figure 1, a disordered electrical activity without the presence of observational QRS and with the peak-to-peak voltage greater than $0.1 \mathrm{mV}$ was annotated as VF. The presence of at least one QRS complex in a segment was classified as PEA. A segment with peak-to-peak voltage less than $0.1 \mathrm{mV}$ was annotated as ASY. Segments with rhythm transitions or defibrillation were excluded from the dataset.

2.2. Estimation of SNR. To investigate the effects of CC on SNRo (before filtering) at different types of underlying rhythms (VF, PEA, and ASY) and performance of the proposed filtering method, we estimate the SNRo and the SNRf (after filtering) of the CPR corrupted ECG based on the contiguous artifact-free signal [27]. Assuming that the underlying ECG and CPR artifact are uncorrelated, the power of CPR artifact can be obtained through subtracting the power of corrupted ECG by the power of clean ECG. Figure 2 shows the examples of signal selection for SNRo estimation in each segment. A 3-second corrupted ECG signal and another 

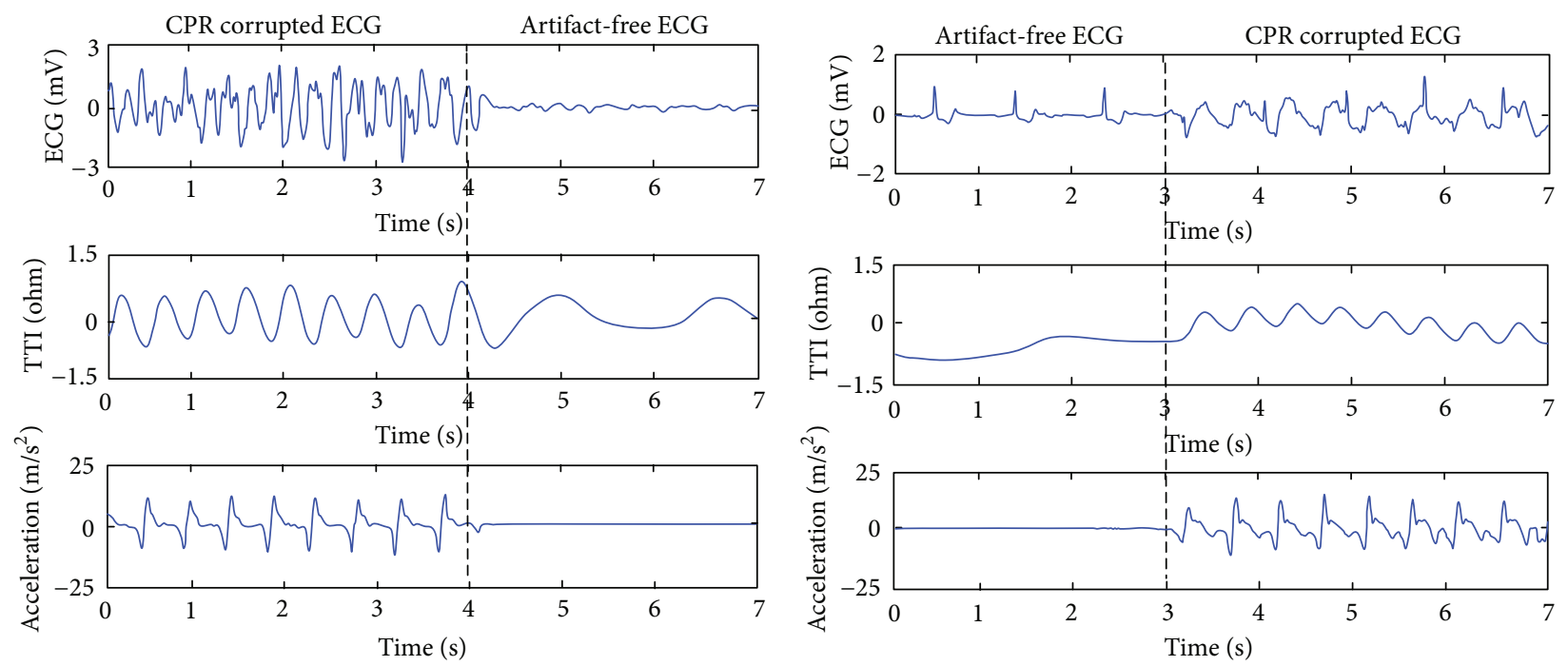

(a)

(b)
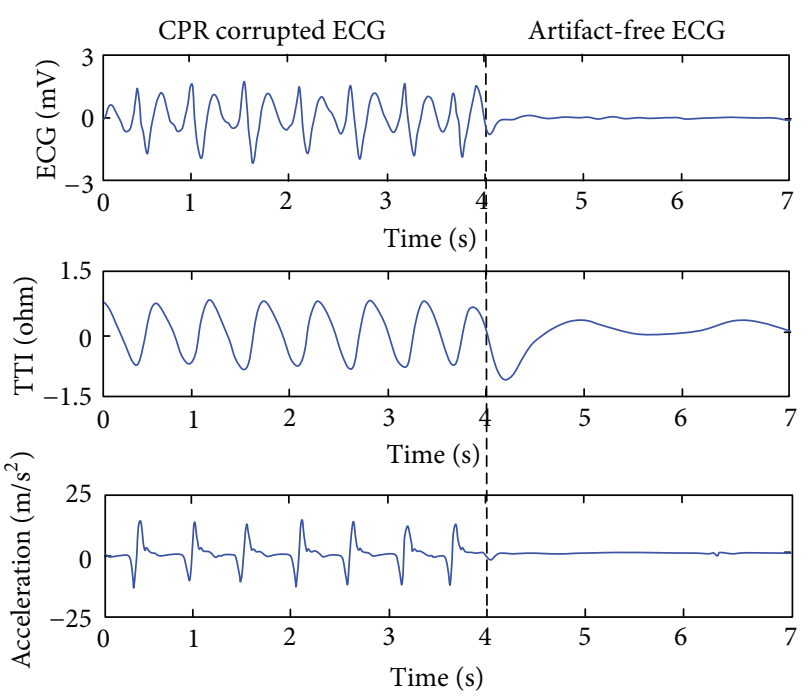

(c)

FIGURE 1: Segments of ECG and reference signals during cardiopulmonary resuscitation (CPR). (a) Ventricular fibrillation with and without chest compression (CC). (b) Pulseless electrical activity (PEA) without and with CC. (c) Asystole (ASY) with and without CC. TTI: transthoracic impedance.

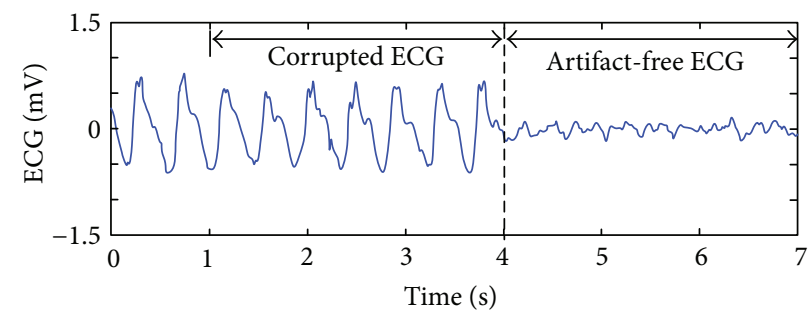

(a)

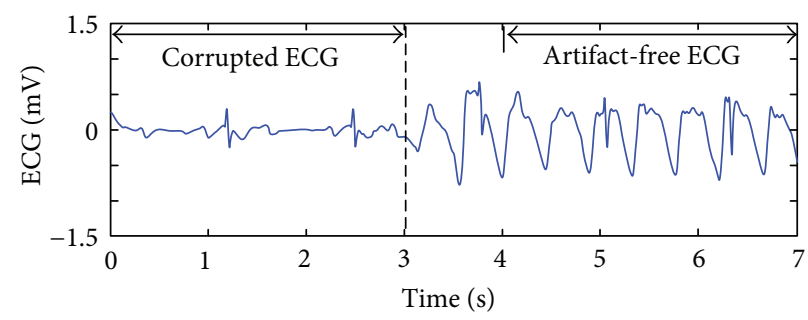

(b)

FIGURE 2: Examples of signal selection for SNR estimation. The CPR corrupted signal was selected either from the latest 3 seconds of chest compression (CC) (a) or 1 second after the beginning of CC (b). 
3-second artifact-free signal are used to calculate the SNRo with the following equation:

$$
\mathrm{SNR}=10 \cdot \log _{10}\left(\frac{\sigma_{s}^{2}}{\sigma_{x}^{2}-\sigma_{s}^{2}}\right),
$$

where $\sigma_{s}^{2}$ is the variance of underlying ECG signal and $\sigma_{x}^{2}$ is the variance of corrupted ECG signal. The SNRf is also estimated with (1), except that the variance of underlying ECG is calculated by the filtered uncorrupted 3-second signal, and the variance of artifact is calculated by the subtraction of the variance of underlying ECG and the variance of filtered corrupted ECG signal.

The estimation is based on the hypothesis that timelimited VF and ASY can be considered quasi-stationary signal. On the other hand, since the energy of a normal sinus rhythm depends on the number of QRS complexes appearing within a segment, we therefore exclude the segments that have unequal numbers of QRS complex within the selected artifact-free and corrupted ECG signals when the underlying rhythm is annotated as PEA.

2.3. The Enhanced Adaptive Filtering Method. To suppress the CC related artifacts (CC-artifact), an enhanced adaptive filtering method is developed by estimating the proportion of artifact within the CPR corrupted ECG signal. The flowchart of the proposed method is shown in Figure 3.

The corrupted ECG and reference (TTI) signals are firstly preprocessed by a 4 th order Butterworth band-pass filter $(0.2-45 \mathrm{~Hz})$ to remove offset and high frequency noise. The power spectral density (PSD) of reference and preprocessed ECG signals are then calculated through dividing the square of the amplitude of fast Fourier transform (FFT) by the length of data points. The frequency of $\mathrm{CC} f_{\mathrm{CC}}$ is obtained by the PSD of TTI:

$$
f_{\mathrm{CC}}=\arg \max _{f} P_{\mathrm{TTI}}(f) .
$$

The power of artifact is computed through the PSD of corrupted ECG with the use of $f_{\mathrm{CC}}$ and its harmonics. The proportion of the artifact power pro is calculated by

$$
\text { pro }=\frac{\sum_{k=1}^{N} P_{S}\left(k \cdot f_{\mathrm{CC}}\right)}{\sum_{f=0}^{f_{S} / 2} P_{S}(f)},
$$

where $k$ is the order of harmonics $(N=3)$ and $f_{s}$ is the sampling rate.

The proportion of the artifact power is then compared with a predefined threshold. If the proportion pro is greater than the preset threshold, the adaptive filter will be applied to the ECG signal to suppress the CPR artifact.

In this enhanced adaptive filtering method, normalized least mean squares (NLMS) is used to adjust the coefficient matrix of adaptive filter, and the step size is dynamically adjusted by the estimated artifact proportion pro:

$$
W(n)=W(n-1)+\frac{\mu \cdot \text { pro }}{\|X\|^{2}} \cdot X(n-1) \cdot e(n) .
$$

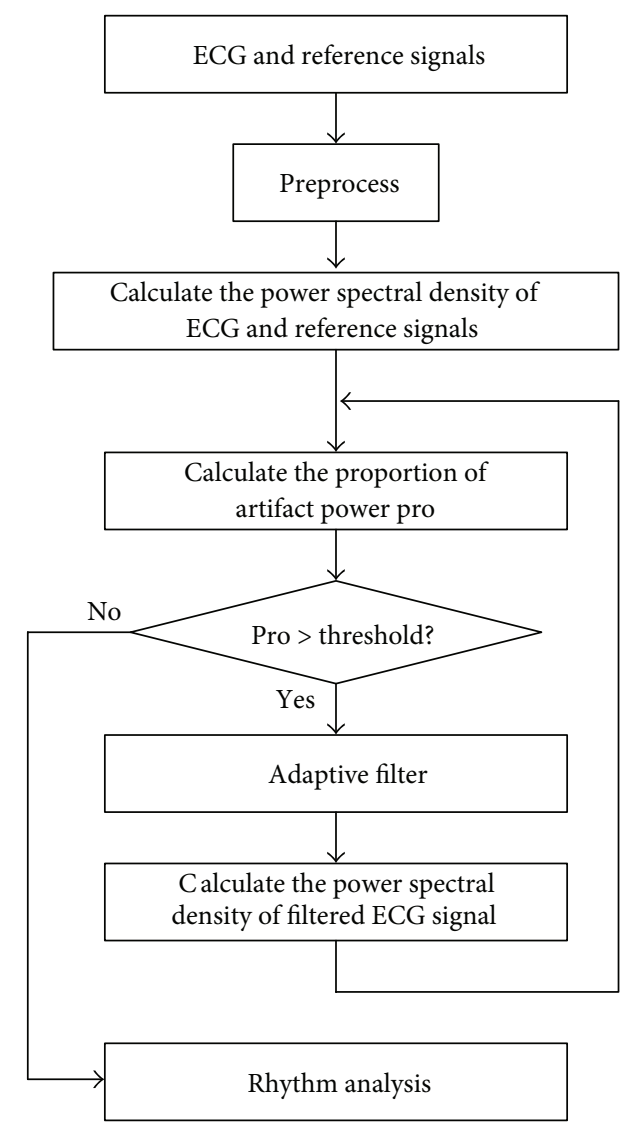

FIgURE 3: Flowchart of the enhanced adaptive filtering method.

The step size $\mu$ is limited by the norm of reference signal $\|X\|$ and proportion of artifact pro. The coefficient matrix $W(n)$ at state $n$ is decided by the previous state $W(n-1)$, the reference signal TTI $X(n-1)$, and the estimated ECG signal $e(n)$ :

$$
e(n)=s_{\text {in }}(n)-W(n) X(n)
$$

where $s_{\text {in }}(n)$ is the input corrupted ECG signal and $W(n) X(n)$ is the estimated CPR artifact.

After filtering, the proportion of artifact pro of the filtered signal is recalculated to assess the SNRf level. If pro is still greater than the preset threshold, another iteration of filtering process will be applied to the filtered signal with updated step size. Otherwise, the filtered ECG signal will be outputted for rhythm analysis. In this study, the length of the coefficient $W(n)$ is 21 , and the step size $\mu$ is 0.15 .

In order to compare the performance with the traditional fixed coefficient high-pass filter [28], a 4th order Butterworth high-pass filter is performed to the corrupted ECG signal to suppress the CPR artifact. Since the average compression rate is $2.11 \mathrm{~Hz}$ in this study, the cutoff frequency is $6.5 \mathrm{~Hz}$ to remove the first 3 harmonics of the artifact.

2.4. Rhythm Classification Algorithm. To evaluate the performance of the proposed method, the sensitivity and specificity for detecting a shockable rhythm before and after filtering 
TABLE 1: Estimated signal-to-noise ratio (SNR) for pulseless electrical activity (PEA), ventricular fibrillation (VF), and asystole (ASY) before and after filtering.

\begin{tabular}{lcrr}
\hline & Unfiltered & Adaptive filter & High-pass filter \\
\hline Medians (dB) (25/75 percentiles) & & & \\
VF & $-9.3(-14.9 /-3.6)^{\Delta \Delta}$ & $0.2(-5.1 / 4.5)^{* *}$ & $0.1(-4.2 / 0.9)^{* *}$ \\
PEA & $-6.2(-9.0 /-1.12)^{\Delta \Delta}$ & $0.1(-3.6 / 3.4)^{* *}$ & $-2.0(-7.4 /-0.6)^{* *}$ \\
ASY & $-21.2(-24.2 /-18.5)^{\Delta \Delta}$ & $-12.7(-15.0 /-4.4)^{* *}$ & $-7.1(-10.7 /-6.3)^{* *}$ \\
Range (dB) (min./max.) & & & $-19.7 / 20.4$ \\
VF & $-26.1 / 9.6$ & $-18.2 / 20.0$ & $-14.0 / 14.7$ \\
PEA & $-16.0 / 9.9$ & $-7.6 / 19.9$ & $-18.4 / 1.7$ \\
ASY & $-31.6 /-10.0$ & $-20.6 / 2.4$ & \\
\hline
\end{tabular}

** Compared with unfiltered signal, $P<0.001 ;{ }^{\triangle}$ comparison among rhythm types, $P<0.001$.

are compared with an established rhythm classification algorithm named phase space reconstruction (RSR) [29, 30]. This specific algorithm is selected because it can provide accurate rhythm classification within a relative short time window. In this method, signal $s(t)$ is plotted on $x$-axis and $s(t+\tau)$ with a delay time of $\tau$ is plotted on $y$-axis to form a two-dimensional phase space diagram. A $40 \times 40$ grid is produced and the number of boxes visited by the signal is counted. Ratio $r^{\prime}$ is calculated through dividing the area that is filled with signal curve $B_{v}$ by the total area of the diagram $B_{a}$. In the current study, the maximum number of data points visited in the box $C_{\max }$ is used to modify the ratio $r^{\prime}$ which is used to classify PEA and VF:

$$
r^{\prime}=\frac{B_{v}}{B_{a}}+\frac{1}{C_{\max }} .
$$

The average peak-to-peak amplitude of the filtered signal $A^{\prime}$ is used to detect ASY. The 3- second ECG signal is split into 3 rectangular nonoverlapping windows. And the difference between maximum and minimum of the signal in each window is calculated and the average of these differences is represented as the value of $A^{\prime}$.

A 3-second rectangular window is used to perform PSR, and the value of $\tau$ is 0.5 seconds. The threshold of the amplitude $A^{\prime}$ and the ratio $r^{\prime}$ are optimized with the artifact-free ECG signals to produce the optimum sensitivity/specificity values. The classification criteria are presented as

$$
\begin{array}{lll}
A^{\prime} \leq 0.1 \mathrm{mV} & \text { ASY } \\
A^{\prime}>0.1 \mathrm{mV}, & r^{\prime} \leq 0.24 & \text { PEA } \\
A^{\prime}>0.1 \mathrm{mV}, & r^{\prime}>0.24 & \text { VF. }
\end{array}
$$

2.5. Statistical Presentation. The distributions of SNRo of the CPR corrupted ECG signal did not pass the KolmogorovSmirnov normality test and were presented as medians (25/75 percentile). The Wilcoxon rank sum test was used for median values comparison. The relationship between SNRo and CD was tested with Pearson correlation coefficients.

The performance of the filtering method was expressed as sensitivity and specificity. Sensitivity and specificity of ECG signals before and after filtering were compared with the classification results of artifact-free ECG signals using Chi-square test. A $P$ value of 0.01 was considered significant.

\section{Results}

The average duration of CPR was $6.8 \pm 3.2$ minutes. A total of 624 segments were extracted and 61 segments were excluded according to the exclusion criteria. Finally, a total of 563 CC related segments, including 283 VF, 208 PEA, and 72 ASY, were obtained for the study. The amplitude of artifact-free ECG signals was $0.7 \pm 0.6 \mathrm{mV}$ for $\mathrm{VF}, 0.8 \pm 0.6 \mathrm{mV}$ for PEA, and $0.05 \pm 0.04 \mathrm{mV}$ for ASY. The amplitude of corrupted ECG signals was $2.1 \pm 1.2 \mathrm{mV}$ for $\mathrm{VF}, 1.9 \pm 0.8 \mathrm{mV}$ for PEA, and $1.0 \pm 0.7 \mathrm{mV}$ for ASY.

3.1. Relationship between CC and SNR. A total of 107 segments of PEA were used for SNR estimation because the numbers of QRS complex within the selected artifact-free and corrupted ECG signals were equal. Table 1 shows the medians (25/75 percentiles) and minimum and maximum value of the estimated SNR based on annotated underlying rhythms. A relative lower SNRo was observed for VF compared with that of PEA $(P<0.001)$ and the SNRo of ASY was significantly lower than PEA and VF $(P<0.001)$. After filtering with the proposed method and high-pass filter, the SNRfs were greatly improved in all of the rhythms $(P<0.001)$.

The linear regression result between SNRo and CD is shown in Figure 4. The SNRo of the full database was negatively correlated with the CD $(r=-0.227, P<0.001)$. When each of the rhythms was investigated individually, negative correlation between CD and SNRo was only observed in VF $(r=-0.239$ and $P<0.001)$.

3.2. Performance of the Enhanced Adaptive Filtering Method. Table 2 shows the rhythm classification results for the artifactfree, CPR corrupted, and filtered signals with the use of PSR. The sensitivity and specificity were $99.0 \%$ and $98.2 \%$ for artifact-free signal. However, the specificity decreased to $46.8 \%$ and the sensitivity increased to $99.3 \%$ when the ECG signals were corrupted by CPR. After filtering by enhanced adaptive filter and high-pass filter, a sensitivity of $93.3 \%$ and $93.0 \%$ and a specificity of $96.0 \%$ and $80.4 \%$ were achieved. 


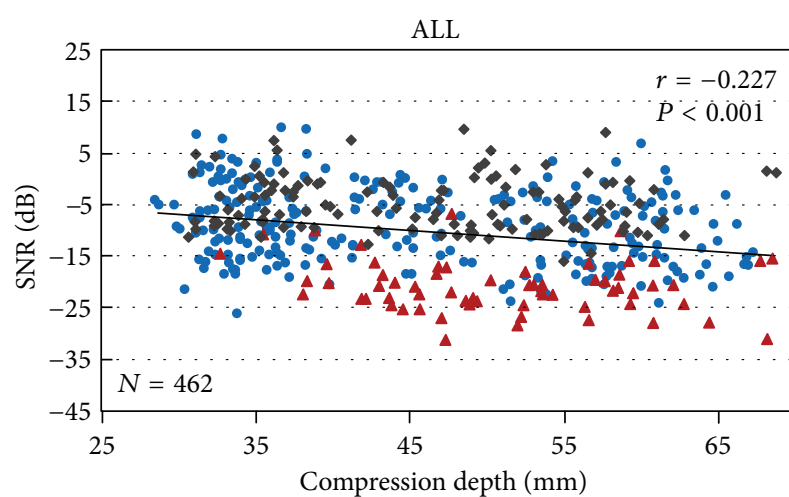

(a)

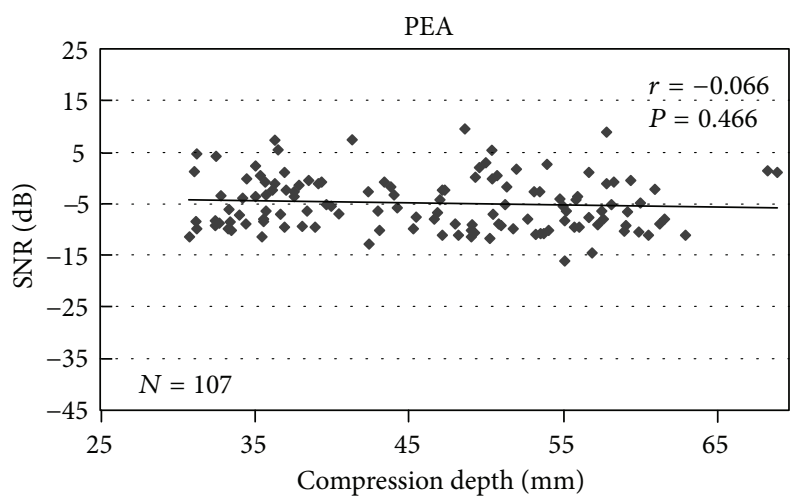

(c)

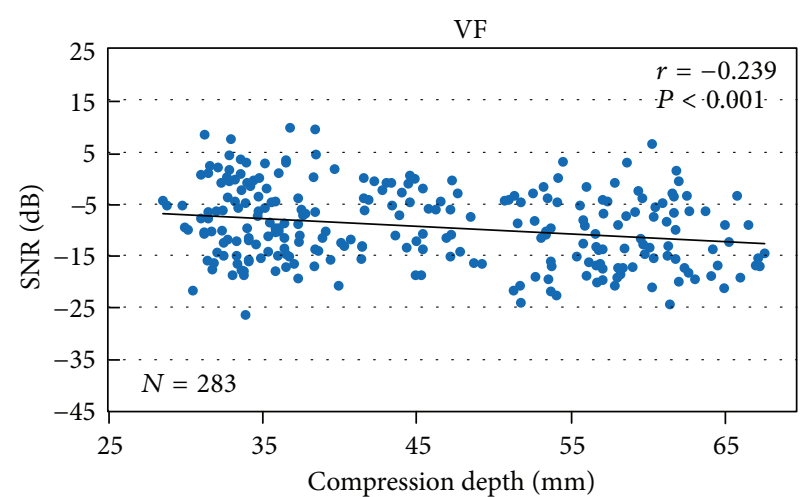

(b)

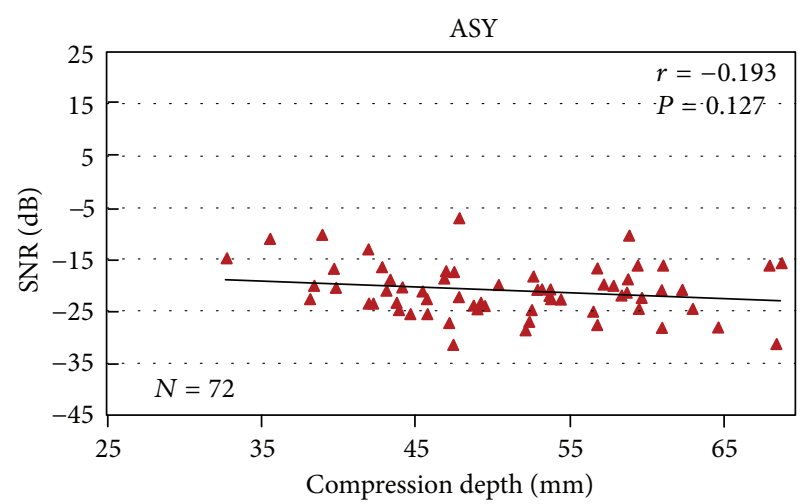

(d)

FIGURE 4: Linear regression results between SNRo and CD for the full database and different types of underlying rhythms (ventricular fibrillation, VF; pulseless electric activity, PEA; asystole, ASY).

TABLE 2: Sensitivity and specificity for the artifact-free ECG and CC corrupted signals before and after filtering.

\begin{tabular}{|c|c|c|c|c|c|c|}
\hline & Rhythm & Number & Artifact-free & Unfiltered & Adaptive & High-pass \\
\hline Shockable (sensitivity) & VF & 283 & $99.0 \%$ & $99.3 \%$ & $93.3 \%{ }^{* *}$ & $93.0 \%{ }^{* *}$ \\
\hline \multirow{3}{*}{ Nonshockable (specificity) } & All & 280 & $98.2 \%{ }^{* *}$ & $46.8 \%$ & $96.0 \%^{* * \# \#}$ & $80.4 \%{ }^{* *}$ \\
\hline & PEA & 208 & $98.6 \%{ }^{* *}$ & $53.9 \%$ & $97.6 \% \%^{* * \# \#}$ & $86.3 \%^{* *}$ \\
\hline & ASY & 72 & $97.2 \%^{* *}$ & $26.4 \%$ & $91.7 \%^{* * \# \#}$ & $63.9 \%{ }^{* *}$ \\
\hline
\end{tabular}

${ }^{* *}$ Compared with unfiltered signals, $P<0.001$ and ${ }^{\# *}$ compared with high-pass filter, $P<0.001$. VF: ventricular fibrillation, PEA: pulseless electrical activity, and ASY: asystole.

\section{Discussion}

The present study confirmed that the SNRo of CPR corrupted ECG was negatively correlated with CD in a porcine model of prolonged cardiac arrest and CPR. Based on this observation, we developed an enhanced adaptive filtering method to suppress the CC-artifact by estimating the proportion of artifact within the corrupted ECG signal. The experimental results demonstrated that the enhanced adaptive filtering method could effectively reduce the residual component of artifact and improve the SNR of the ECG signal as well as the outcome of specificity.

4.1. Relationship between CC and SNR. The CC-artifact was predominant from the electrode-skin interface and generated by the contraction of thoracic muscles with direct impact of the compressions on chest wall [31]. Therefore, it was anticipated that deeper compression would cause more chest movements and introduced severe artifact to the ECG. In this animal study, we demonstrated that the SNRo of CPR corrupted ECG signals was negatively related to CD. However, when each of the rhythm was investigated, the SNRo was significantly lower for ASY compared to PEA and VF and the negative correlation between CD and SNRo was only observed in VF. For VF, the signal energy homogeneously distributed among all VF segments, and the value of SNRo was therefore correlated with the value of CD. For PEA, the energy of underlying signal depended on the number of QRS complexes appearing within a segment and might impact the correlation between SNRo and CD. For ASY, the energy of underlying signal is theoretically nearly 0 so that the SNRo should be $-\infty$. However, randomized noisy signal 
and power supply artifact, together with artifacts produced by the amplifier and $\mathrm{A} / \mathrm{D}$ converter, were introduced during measurement. Even though a band-pass filter was applied before analysis, the irregular residual signals within underlying ASY might still affect the value of signal energy and lead to insignificant correlation between SNRo and CD.

Compared with the result that was reported by de Gauna et al. [27], a relatively lower SNRo was observed in our study. The inconsistence may relate to the increased CD recommendation of the latest guidelines, which require a minimum of $50 \mathrm{~mm}$ in $\mathrm{CD}$ to ensure high quality CPR [10, 32]. At the same time, signal characteristics of porcine ECG such as amplitude and frequency might be different from that of human. The resulted SNRo thus would be affected by the spectral energy calculated from signal amplitude and frequency.

\subsection{Improved Performance for the Enhanced Adaptive Filtering} Method. Based on the findings that SNRo was negatively correlated with $\mathrm{CD}$, we developed an enhanced adaptive filtering method to suppress the CPR artifact by estimating the proportion of artifact with the use of TTI as reference. Compared with the corrupted signal, both traditional fixed coefficient high-pass filter and proposed method could greatly improve the SNR and specificity. But compared with a specificity of $80.4 \%$ for high-pass filter, a remarkable improvement was achieved for the proposed method with a value of $96.0 \%$.

The following modification in removing the CPR related artifact might contribute to the improved performance of the proposed method. Firstly, a parameter was introduced to estimate the proportion of artifact from PSD of ECG signal with the use of compression frequency as reference. The proportion of artifact was correlated with the power of artifact and therefore the SNR level. Secondly, the step size of commonly used LMS adaptive filter was dynamically adjusted by referenced TTI signal and the estimated proportion of artifact. This modification provided greater stability and convergence speed compared with traditional LMS based adaptive method which was used by Irusta et al. [17] and Aramendi et al. [18]. Therefore, the specificity of the proposed method was greatly improved compared with their results even though similar reference signals were used in both studies. Thirdly, the proportion of artifact was also used as an indicator to assess the artifact level in the filtered signal and to control the filtering iteration. This process was terminated only if the artifact level decreased to a predefined threshold. Compared with the MC-RAMP method which took use of several kinds of reference signals proposed by Husøy et al. [33] and Eilevstjønn et al. [20], the residual component of artifact could be further suppressed and the reliability for detecting a nonshockable rhythm was markedly improved.

Besides the enhanced adaptive filter, the algorithm used for rhythm classification also contributed to the improved specificity. The parameters were optimized according to clean ECG signals recorded from the animals when SPR was used [29]. Firstly, the ratio $r^{\prime}$ was adjusted by the maximum number of data points visited in the box. This adjustment enlarged the difference between VF and PEA. Secondly, both window size and delay time were optimized when the phase space diagram was reconstructed. Consequently, the threshold of $r^{\prime}$ increased from 0.15 to 0.24 for the detection of VF.

Although the SNR and specificity were greatly improved after filtering, the sensitivity decreased from $99.3 \%$ to $93.3 \%$. It is because the enhanced filtering method also suppressed the component of underlying ECG signals while removing the CPR related artifact. As a result, amplitude of fine VF might be reduced to a level that is below the criteria for classification. When the nonshockable rhythms were investigated separately, the specificity for detecting ASY was relatively lower compared with that of PEA and still below the 95\% limit recommended by AHA task force on AEDs [22]. This was consistent with the observation that CPR artifact suppression was particularly difficult in ASY [34, 35]. Yet, the $91.7 \%$ specificity for detecting ASY was still superior to reported results and the adverse effects of interruption of CC are likely to override the decrease in correctly detecting ASY.

4.3. Limitations. There are limitations that need to be acknowledged and addressed regarding the present study. Firstly, although the SNRo of CPR corrupted ECG was demonstrated to be negatively correlated with $\mathrm{CD}$ for the full database, this correlation was only observed in VF when different ECG rhythms were investigated individually. Additionally, the anatomy structure of human chest was different with that of the animals. Therefore the relationship between artifact level and CD in human beings at different underlying rhythms is still needed to be investigated. Secondly, only TTI signal was used as reference in this study; the effects of different reference signals on the performance of the proposed method have not been investigated. Thirdly, although a great improvement in specificity was achieved in this experimental trial, characteristics of ECG waveform, together with the CPR related artifact, may differ from the data that are recorded from patients who experienced out-of-hospital cardiac arrest and CPR. Performance of the proposed method therefore needs further clinical validating studies. Finally, even though the specificity for detecting a nonshockable rhythm was greatly improved and above the $95 \%$ limit recommended by the AHA task force on AEDs [22], the accuracy for detecting ASY was still low. Further studies that focused on the suppressing artifact of ASY, as well as the classification between ASY and VF, still need to be conducted.

\section{Conclusion}

This experimental animal trial demonstrated that the SNRo of ECG signal corrupted by CPR artifact was negatively correlated with $\mathrm{CD}$ and the enhanced adaptive filtering method could significantly improve the detection of nonshockable rhythms without compromising the ability to detect a shockable rhythm during uninterrupted CPR.

\section{Conflict of Interests}

The authors declare that there is no conflict of interests regarding the publication of this paper. 


\section{Acknowledgments}

This study was supported in part by the National Nature Science Foundation of China (NSFC81271656), a foundation from the General Logistics Department of PLA (CWS12J094), and a Foundation for the Author of National Excellent Doctoral Dissertation of China (FANEDD 201060).

\section{References}

[1] T. D. Valenzuela, D. J. Roe, S. Cretin, D. W. Spaite, and M. P. Larsen, "Estimating effectiveness of cardiac arrest interventions: a logistic regression survival model," Circulation, vol. 96, no. 10, pp. 3308-3313, 1997.

[2] T. D. Valenzuela, D. J. Roe, G. Nichol, L. L. Clark, D. W. Spaite, and R. G. Hardman, "Outcomes of rapid defibrillation by security officers after cardiac arrest in casinos," The New England Journal of Medicine, vol. 343, no. 17, pp. 1206-1209, 2000.

[3] A. P. van Alem, B. T. Sanou, and R. W. Koster, "Interruption of cardiopulmonary resuscitation with the use of the automated external defibrillator in out-of-hospital cardiac arrest," Annals of Emergency Medicine, vol. 42, no. 4, pp. 449-457, 2003.

[4] T. Eftestøl, K. Sunde, and P. A. Steen, "Effects of interrupting precordial compressions on the calculated probability of defibrillation success during out-of-hospital cardiac arrest," Circulation, vol. 105, no. 19, pp. 2270-2273, 2002.

[5] T. Yu, M. H. Weil, W. Tang et al., "Adverse outcomes of interrupted precordial compression during automated defibrillation," Circulation, vol. 106, no. 3, pp. 368-372, 2002.

[6] T. D. Valenzuela, K. B. Kern, L. L. Clark et al., "Interruptions of chest compressions during emergency medical systems resuscitation," Circulation, vol. 112, no. 9, pp. 1259-1265, 2005.

[7] D. P. Edelson, B. S. Abella, J. Kramer-Johansen et al., "Effects of compression depth and pre-shock pauses predict defibrillation failure during cardiac arrest," Resuscitation, vol. 71, no. 2, pp. 137-145, 2006.

[8] K. Gundersen, J. T. Kvaløy, J. Kramer-Johansen, P. A. Steen, and T. Eftestøl, "Development of the probability of return of spontaneous circulation in intervals without chest compressions during out-of-hospital cardiac arrest: an observational study," BMC Medicine, vol. 7, article 6, 2009.

[9] S. Cheskes, R. H. Schmicker, J. Christenson et al., "Perishock pause: an independent predictor of survival from out-ofhospital shockable cardiac arrest," Circulation, vol. 124, no. 1, pp. 58-66, 2011.

[10] M. S. Link, D. L. Atkins, R. S. Passman et al., "Part 6: electrical therapies: automated external defibrillators, defibrillation, cardioversion, and pacing: 2010 American Heart Association guidelines for cardiopulmonary resuscitation and emergency cardiovascular care," Circulation, vol. 122, no. 18, pp. S706-S719, 2010.

[11] C. D. Deakin, J. P. Nolan, K. Sunde, and R. W. Koster, "European resuscitation council guidelines for resuscitation 2010 section 3. Electrical therapies: automated external defibrillators, defibrillation, cardioversion and pacing," Resuscitation, vol. 81, no. 10, pp. 1293-1304, 2010.

[12] M. Granegger, T. Werther, and H. Gilly, "Use of independent component analysis for reducing CPR artefacts in human emergency ECGs," Resuscitation, vol. 82, no. 1, pp. 79-84, 2011.

[13] A. Amann, A. Klotz, T. Niederklapfer et al., "Reduction of CPR artifacts in the ventricular fibrillation ECG by coherent line removal," BioMedical Engineering Online, vol. 9, article 2, 2010.
[14] T. Werther, A. Klotz, G. Kracher et al., "CPR artifact removal in ventricular fibrillation ECG signals using gabor multipliers," IEEE Transactions on Biomedical Engineering, vol. 56, no. 2, pp. 320-327, 2009.

[15] K. Rheinberger, T. Steinberger, K. Unterkofler, M. Baubin, A. Klotz, and A. Amann, "Removal of CPR artifacts from the ventricular fibrillation ECG by adaptive regression on lagged reference signals," IEEE Transactions on Biomedical Engineering, vol. 55, no. 1, pp. 130-137, 2008.

[16] A. Langhelle, T. Eftestol, H. Myklebust, M. Eriksen, B. T. Holten, and P. A. Steen, "Reducing CPR artefacts in ventricular fibrillation in vitro," Resuscitation, vol. 48, no. 3, pp. 279-291, 2001.

[17] U. Irusta, J. Ruiz, S. R. de Gauna, T. Eftestøl, and J. KramerJohansen, "A least mean-Square filter for the estimation of the cardiopulmonary resuscitation artifact based on the frequency of the compressions," IEEE Transactions on Biomedical Engineering, vol. 56, no. 4, pp. 1052-1062, 2009.

[18] E. Aramendi, U. Ayala, U. Irusta, E. Alonso, T. Eftestøl, and J. Kramer-Johansen, "Suppression of the cardiopulmonary resuscitation artefacts using the instantaneous chest compression rate extracted from the thoracic impedance," Resuscitation, vol. 83, no. 6, pp. 692-698, 2012.

[19] S. O. Aase, T. Eftestol, J. H. Husoy, K. Sunde, and P. A. Steen, "CPR artifact removal from human ECG using optimal multichannel filtering," IEEE Transactions on Biomedical Engineering, vol. 47, no. 11, pp. 1440-1449, 2000.

[20] J. Eilevstjønn, T. Eftestøl, S. O. Aase, H. Myklebust, J. H. Husøy, and P. A. Steen, "Feasibility of shock advice analysis during CPR through removal of CPR artefacts from the human ECG," Resuscitation, vol. 61, no. 2, pp. 131-141, 2004.

[21] Y. Li, J. Bisera, F. Geheb, W. Tang, and M. H. Weil, "Identifying potentially shockable rhythms without interrupting cardiopulmonary resuscitation," Critical Care Medicine, vol. 36, no. 1, pp. 198-203, 2008.

[22] R. E. Kerber, L. B. Becker, J. D. Bourland et al., "Automatic external defibrillators for public access defibrillation: recommendations for specifying and reporting arrhythmia analysis algorithm performance, incorporating new waveforms, and enhancing safety. A statement for health professionals from the American Heart Association Task Force on automatic external defibrillation subcommittee on AED safety and efficacy," Circulation, vol. 95, no. 6, pp. 1677-1682, 1997.

[23] Y. Li and W. Tang, "Techniques for artefact filtering from chest compression corrupted ECG signals: good, but not enough," Resuscitation, vol. 80, no. 11, pp. 1219-1220, 2009.

[24] Y. Gong, B. Chen, and Y. Li, "A review of the performance of artifact filtering algorithms for cardiopulmonary resuscitation," Journal of Healthcare Engineering, vol. 4, no. 2, pp. 185-202, 2013.

[25] W. G. Grundler, M. H. Weil, J. M. Miller, and E. C. Rackow, "Observations on colloid osmotic pressure, hematocrit, and plasma osmolality during cardiac arrest," Critical Care Medicine, vol. 13, no. 11, pp. 895-896, 1985.

[26] H. Li, L. Zhang, Z. Yang et al., "Even four minutes of poor quality of CPR compromises outcome in a porcine model of prolonged cardiac arrest," BioMed Research International, vol. 2013, Article ID 171862, 6 pages, 2013.

[27] S. R. de Gauna, J. Ruiz, U. Irusta, and U. Ayala, "Filtering the cardiopulmonary resuscitation artifact: influence of the signalto-noise-ratio on the accuracy of the shock advice algorithm," in Proceedings of the Computing in Cardiology (CinC '10), vol. 37, pp. 681-684, September 2010. 
[28] H.-U. Strohmenger, K. H. Lindner, A. Keller, I. M. Lindner, and E. G. Pfenninger, "Spectral analysis of ventricular fibrillation and closed-chest cardiopulmonary resuscitation," Resuscitation, vol. 33, no. 2, pp. 155-161, 1996.

[29] A. Amann, R. Tratnig, and K. Unterkofler, "A new ventricular fibrillation detection algorithm for automated external defibrillators," in Proceedings of the Computers in Cardiology, pp. 559562, Lyon, France, September 2005.

[30] A. Amann, R. Tratnig, and K. Unterkofler, "Detecting ventricular fibrillation by time-delay methods," IEEE Transactions on Biomedical Engineering, vol. 54, no. 1, pp. 174-177, 2007.

[31] E. Fitzgibbon, R. Berger, J. Tsitlik, and H. R. Halperin, "Determination of the noise source in the electrocardiogram during cardiopulmonary resuscitation," Critical Care Medicine, vol. 30, supplement 4, pp. S148-S153, 2002.

[32] A. H. Travers, T. D. Rea, B. J. Bobrow et al., "Part 4: CPR overview: 2010 American Heart Association guidelines for cardiopulmonary resuscitation and emergency cardiovascular care," Circulation, vol. 122, no. 18, pp. S676-S684, 2010.

[33] J. H. Husøy, J. Eilevstjønn, T. Eftestøl, S. O. Aase, H. Myklebust, and P. A. Steen, "Removal of cardiopulmonary resuscitation artifacts from human ECG using an efficient matching pursuitlike algorithm," IEEE Transactions on Biomedical Engineering, vol. 49, no. 11, pp. 1287-1298, 2002.

[34] T. Werther, A. Klotz, M. Granegger et al., "Strong corruption of electrocardiograms caused by cardiopulmonary resuscitation reduces efficiency of two-channel methods for removing motion artefacts in non-shockable rhythms," Resuscitation, vol. 80, no. 11, pp. 1301-1307, 2009.

[35] S. Ruiz de Gauna, J. Ruiz, U. Irusta, E. Aramendi, T. Eftestøl, and J. Kramer-Johansen, "A method to remove CPR artefacts from human ECG using only the recorded ECG," Resuscitation, vol. 76, no. 2, pp. 271-278, 2008. 


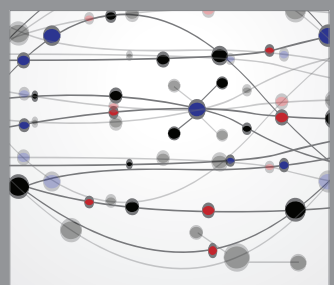

The Scientific World Journal
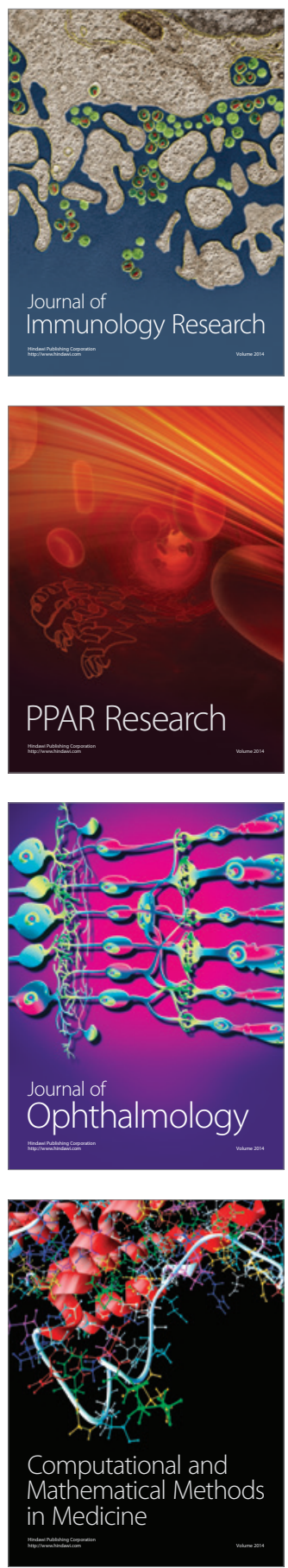

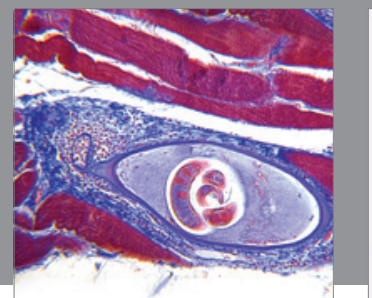

Gastroenterology

Research and Practice
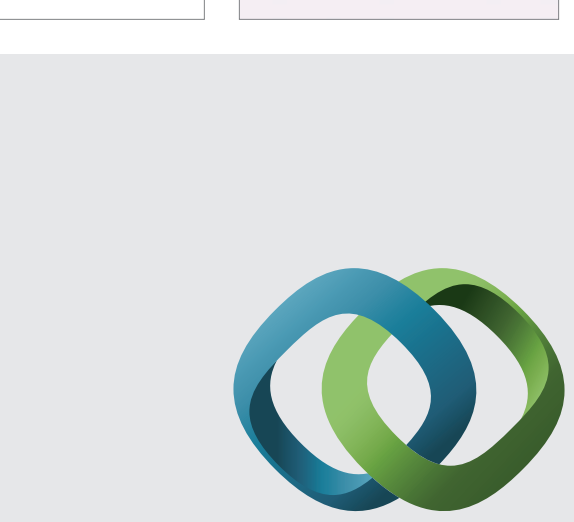

\section{Hindawi}

Submit your manuscripts at

http://www.hindawi.com
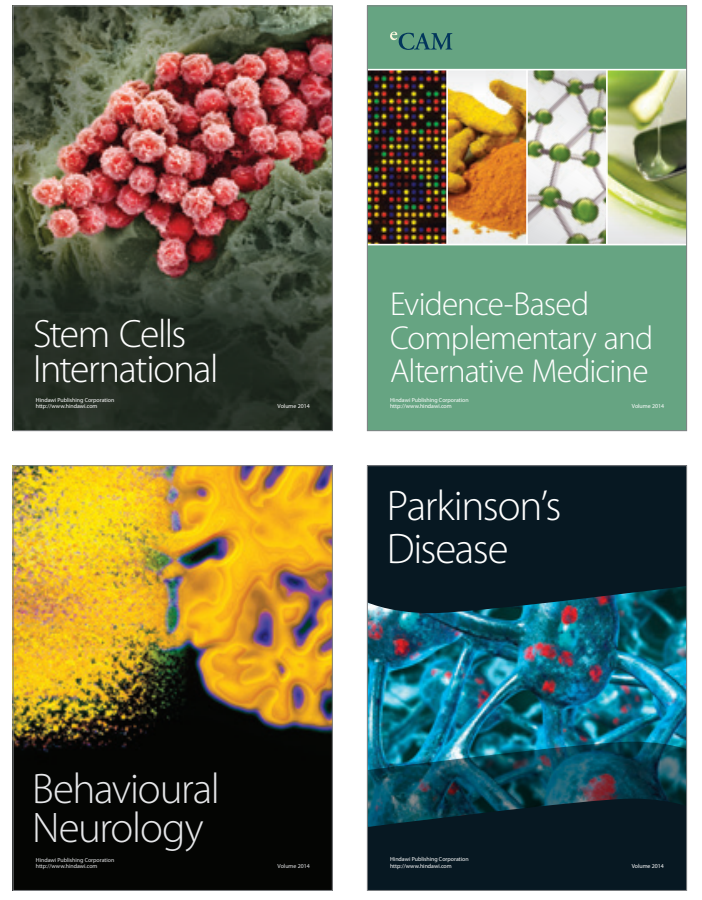
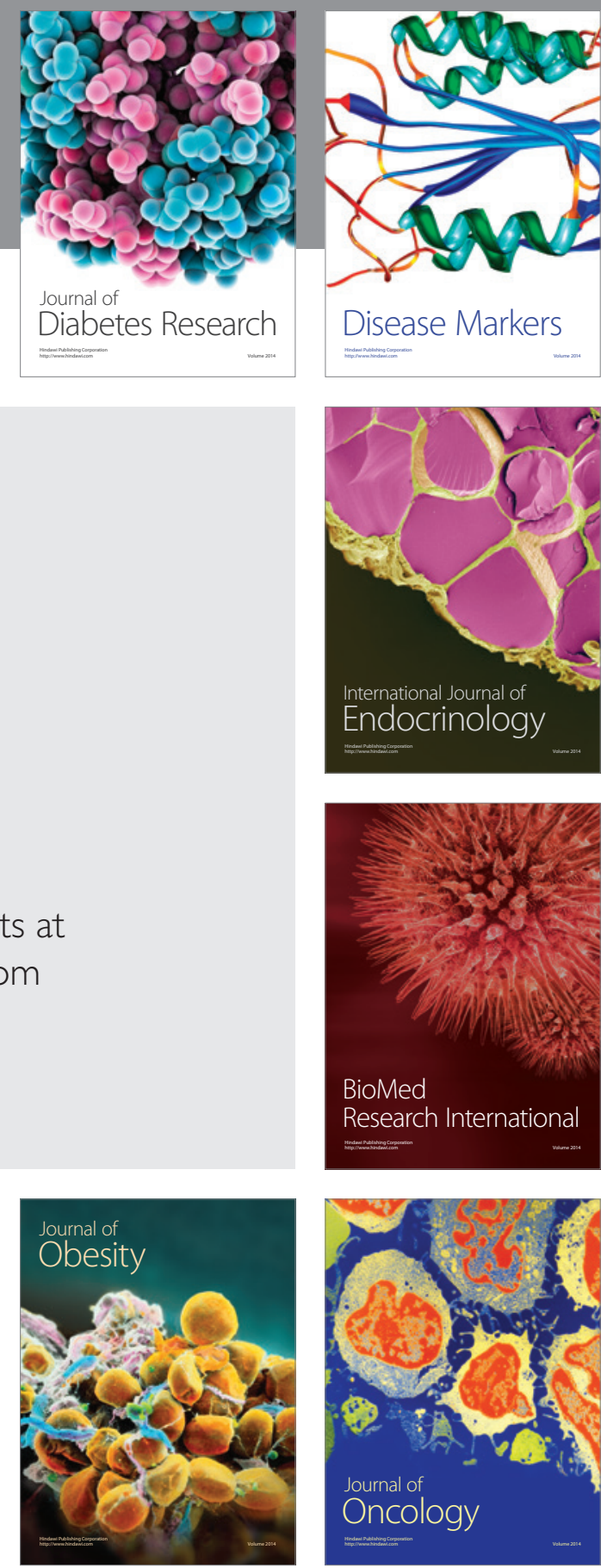

Disease Markers
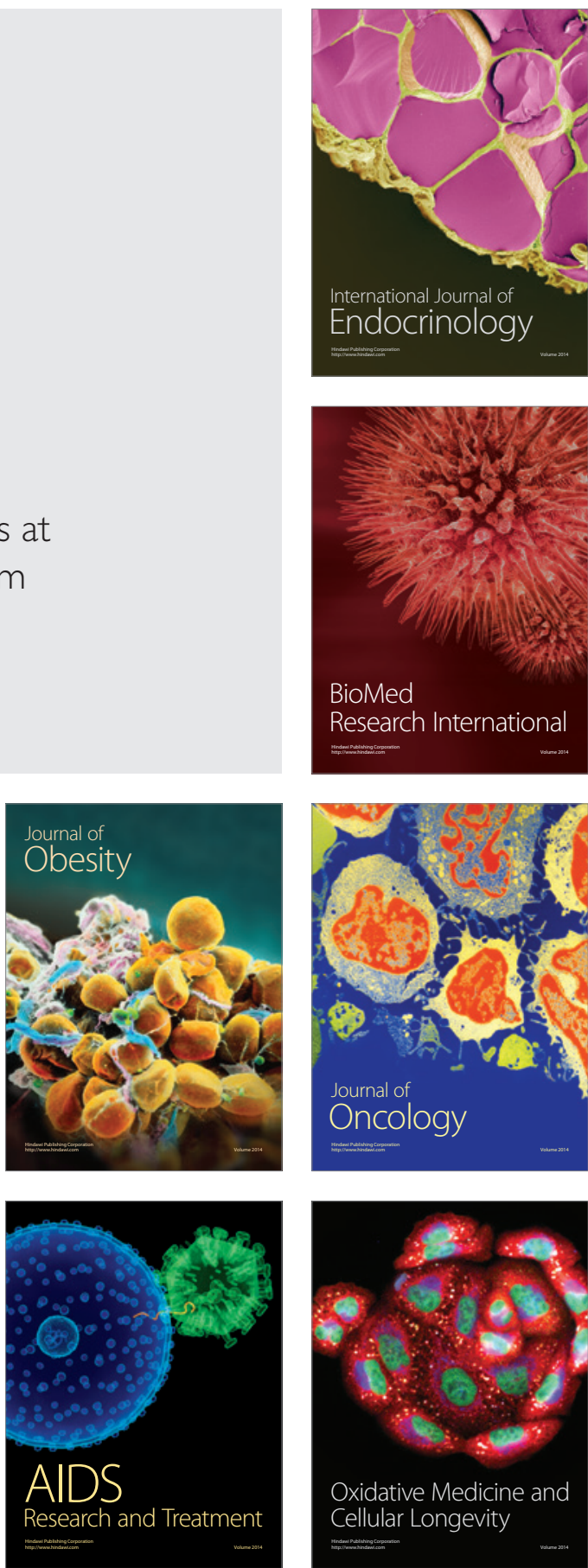